Joleen Hubbard, Erin Green, Qian Shi, and Daniel Sargent, Mayo Clinic, Rochester, MN; David M. Thomas, Peter MacCallum Cancer Centre, East Melbourne, Australia; Greg Yothers and Michael J. O'Connell, National Surgical Adjuvant Breast and Bowel Project; Greg Yothers, University of Pittsburgh, Pittsburgh, PA; Charles Blanke, British Columbia Cancer Agency and University of British Columbia, Vancouver, British Columbia, Canada; Roberto Labianca, Ospedali Riuniti, Bergamo, Italy; Archie Bleyer, St Charles Health System, Bend, OR; and Aimery de Gramont, Hopital Saint Antoine, Paris, France.

This was a joint project of the LIVESTRONG Young Adult Alliance and the Adjuvant Colon Cancer Endpoints (ACCENT) Group. Full group membership is listed in the Appendix (online only).

Submitted December 13, 2011: accepted February 28, 2012; published online ahead of print at www.jco.org on May 21, 2012

Supported by National Cancer Institute Grants No. CA25225, CA12027, CA69974, CA37377, CA69651, and CA114732 and the Livestrong Young Adult Alliance.

Authors' disclosures of potential conflicts of interest and author contributions are found at the end of this article.

Corresponding author: Daniel Sargent, $\mathrm{PhD}$, Division of Biomedical Statistics and Informatics, Harwick 8-27, 200 First St Southwest, Rochester, MN 55905 e-mail: sargent.daniel@mayo.edu.

(ㄷ) 2012 by American Society of Clinical Oncology

0732-183X/12/3019-2334/\$20.00

DOI: 10.1200/JCO.2011.41.1975

\title{
Benefits and Adverse Events in Younger Versus Older Patients Receiving Adjuvant Chemotherapy for Colon Cancer: Findings From the Adjuvant Colon Cancer Endpoints Data Set
}

Joleen Hubbard, David M. Thomas, Greg Yothers, Erin Green, Charles Blanke, Michael J. O'Connell, Roberto Labianca, Qian Shi, Archie Bleyer, Aimery de Gramont, and Daniel Sargent

$$
\begin{array}{llllllll}
\text { A } & \text { B } & \text { S } & \text { T } & \text { R } & \text { A } & \text { C } & \text { T }
\end{array}
$$

\section{Purpose}

Limited data exist regarding the outcomes of adjuvant therapy in younger patients with stage II and III colon cancer. We examined disease-free survival (DFS), overall survival (OS), recurrencefree interval (RFI), and grade 3+ adverse events (AEs) in younger patients in the 33,574 patient Adjuvant Colon Cancer Endpoints Group data set.

\section{Patients and Methods}

Individual patient data from 24 randomized phase III clinical trials were obtained for survival outcomes, which included 10 clinical trials for AE outcomes. Two age-based cutoff points were used to define younger patients: age younger than 40 years and younger than 50 years. Adjuvant therapy benefit analyses were limited to the nine clinical trials in which the investigational chemotherapeutic arm demonstrated benefit.

\section{Results}

One thousand seven hundred fifty-eight patients (5.2\%) were younger than 40 years, 5,817 patients (17.3\%) were younger than 50 years, and only 299 patients $(0.9 \%)$ were younger than 30 years. No meaningful differences in sex or stage were noted in younger versus older patients. Younger and older patients did not differ in RFI (age, $<40$ years: hazard ratio [HR], $1.0 ; P=.62$ and age $<50$ years: HR, 1.02; $P=.35$ ). Younger patients (both cutoff points), had longer OS and DFS than older patients. In trials demonstrating adjuvant therapy benefit, similar DFS benefit was observed by age. Younger patients experienced less leukopenia and stomatitis, but more frequent nausea/vomiting.

\section{Conclusion}

Among patients on clinical trials, younger and older patients with stage II and III colon cancer had similar RFI and adjuvant therapy benefit. Younger patients have longer OS and DFS, which is likely primarily because of fewer competing causes of death. Adjuvant therapy is beneficial for colon cancer in patients younger than 50 years who meet typical clinical trial eligibility criteria.

\section{J Clin Oncol 30:2334-2339. (C) 2012 by American Society of Clinical Oncology}

\section{INTRODUCTION}

Cancer is the second leading cause of disease-related death among those age 20 to 39 years. $^{1}$ Although advances in cancer-related mortality have been made in children and older adults over the last two decades, similar progress has not been made in the young adult population, regardless of sex or ethnicity. ${ }^{2,3}$ The reasons for the lack of progress are unclear and difficult to investigate owing to the lack of this age group's participation in clinical trials. ${ }^{4}$ As a result, little is known about the optimal treatments and outcomes for adolescents and young adults with cancer.
To address this knowledge deficit, the National Cancer Institute partnered with the Lance Armstrong Foundation to form the Adolescent and Young Adult Oncology Progress Review Group (AYAO PRG). This group developed a national agenda to improve cancer prevention, early detection, diagnosis, treatment, and outcomes of adolescents and young adults. ${ }^{5}$ Among the recommendations was a call for the better understanding of disease outcomes and the response to treatment for younger patients when compared with older patients. Colorectal cancer (CRC) was identified as one of the more frequent cancers in this age group, thus warranting further investigation. ${ }^{6}$ 


\begin{tabular}{|ccc|}
\hline \multicolumn{3}{|c|}{ Table 1. Distribution of Patients by Age } \\
\hline Patient Age (years) & No. of Patients & $\%$ \\
\hline$<30$ & 299 & 0.9 \\
$30-34$ & 503 & 1.5 \\
$35-39$ & 956 & 2.9 \\
$40-44$ & 1,549 & 4.6 \\
$45-49$ & 2,510 & 7.4 \\
$50-54$ & 3,818 & 11.4 \\
$55-59$ & 5,056 & 15.1 \\
$60-64$ & 6,188 & 18.4 \\
$65-69$ & 6,610 & 19.7 \\
$70-74$ & 4,488 & 13.4 \\
$75-79$ & 1,334 & 3.9 \\
$\geq 80$ & 263 & 0.8 \\
Total & 33,574 & 100 \\
\hline
\end{tabular}

CRC is the third leading cause of cancer death among men and fourth leading cause of cancer death among women, ages 20 to 39 years. ${ }^{1}$ Among patients age 15 to 29 years, nonstage adjusted survival rates for CRC are lower than those of their older adult counterparts, which is likely related to the higher frequency of stage III and IV CRC at presentation. ${ }^{7,8}$ When matched for stage, survival rates seem to be similar or better for young adults compared with older adults. ${ }^{8,9}$ However, little is known about toxicities and benefits of adjuvant therapy in young adults.

This study evaluates outcomes in young adults with colon cancer using the Adjuvant Colon Cancer Endpoints (ACCENT) database. ACCENT is a pooled database of data on individual patients with stage I to III colon cancer from multiple phase III clinical trials involving fluorouracil (FU) -based adjuvant therapy. Initially established in $2003^{10}$ and updated in $2009,{ }^{11}$ the goal of this international collaboration is to understand the benefits of adjuvant therapy for stage II and stage III colon cancer and develop optimal standards for clinical trial design. We sought to determine the disease outcomes, benefits of therapy, and types of adverse events among younger patients within the ACCENT database.

\section{PATIENTS AND METHODS}

The ACCENT data set consists of individual patient data from 24 large randomized trials that investigated adjuvant therapy in colon cancer. These trials, conducted from 1978 to 2003 , involved 33,574 patients and 55 treatment arms (46 active treatment arms and nine surgery-only arms). Details regarding the individual clinical trials are provided in the Appendix (online-only). Specific data on drug dosing are not available. Approval for this analysis was granted by the Mayo Clinic Investigational Review Board; individual trials were approved through local mechanisms at the time the trials were conducted.

Young patients were defined to be younger than 40 years of age at time of random assignment per the AYAO PRG recommendations. A separate analysis was performed using 50 years as a cutoff age because of sex-related differences in toxicity among younger patients receiving FU therapy and the timing of menopause. ${ }^{12}$ The primary end point for the analysis was disease-free survival (DFS), defined as time from randomization to disease recurrence or death as a result of any cause. Secondary end points were overall survival (OS), recurrence-free interval (RFI; defined as time to disease recurrence; patients who died without recurrence have their RFI time censored at time of death), and adverse events (AEs) grade 3 or higher based on Common Toxicity Criteria used with each of the individual trials. From a clinical perspective RFI is a pure tumor-only end point, because patients who die as a result of nondisease factors are not considered as events in the analysis. Comparisons of raw (as opposed to relative) DFS, RFI, and OS were used as the primary analysis methodology, as the patients in the ACCENT database are all from clinical trials and likely differ in many aspects from community-based cohorts, thus making relative survival difficult to interpret in this context. Cox regression models stratified by study and adjusted for sex and stage were used to evaluate the effect of age on survival outcomes. Logistic regression models adjusting for study, sex, and stage were used to evaluate the impact of age on AEs. In addition, the associations between age and survival outcomes and treatment toxicities were examined separately within stage II and III patients.

Within the ACCENT database, nine clinical trials demonstrated a benefit of adjuvant therapy, seven clinical trials tested FU combined with leucovorin or levamisole (FU/LV) versus control and two trials compared oxaliplatin with $\mathrm{FU} / \mathrm{LV}$ versus FU/LV alone. Additional analyses were performed on these nine trials to evaluate whether the treatment effect was different between younger and older patients, testing the interaction effect between age group and treatment assignment using a likelihood ratio test in the Cox regression analysis. OS outcomes were compared descriptively to the age-matched United States white population, with the relative survival rate defined as the observed 5-year survival rate divided by the age-matched expected 5-year survival rate among the United States white population.

\section{RESULTS}

The age distribution and baseline characteristics of included patients by age group are listed in Tables 1 and 2, respectively. One thousand seven hundred fifty-eight patients (5.2\%) were younger than 40 years; 5,817 patients $(17.3 \%)$ were younger than 50 years. Only 299 patients $(0.9 \%)$ were younger than 30 years. Statistically significant but clinically modest differences in sex and stage between younger and older

\begin{tabular}{|c|c|c|c|c|c|c|c|c|c|c|}
\hline \multirow[b]{2}{*}{ Characteristic } & \multicolumn{2}{|c|}{$\begin{array}{c}\text { Age }<40 \text { Years } \\
(n=1,758)\end{array}$} & \multicolumn{2}{|c|}{$\begin{array}{c}\text { Age } \geq 40 \text { Years } \\
(n=31,816)\end{array}$} & \multirow[b]{2}{*}{$P$} & \multicolumn{2}{|c|}{$\begin{array}{c}\text { Age }<50 \text { Years } \\
(n=5,817)\end{array}$} & \multicolumn{2}{|c|}{$\begin{array}{c}\text { Age } \geq 50 \text { Years } \\
(n=27,757)\end{array}$} & \multirow[b]{2}{*}{$P$} \\
\hline & No. of Patients & $\%$ & No. of Patients & $\%$ & & No. of Patients & $\%$ & No. of Patients & $\%$ & \\
\hline Sex & & & & & & & & & & $<.0001$ \\
\hline Male & 923 & 53 & 17,436 & 55 & & 2,985 & 51 & 15,374 & 55 & \\
\hline Female & 833 & 48 & 14,378 & 45 & .07 & 2,830 & 49 & 12,381 & 45 & \\
\hline Stage & & & & & & & & & & $<.0001$ \\
\hline I & 5 & 0.3 & 217 & 0.7 & & 17 & 0.3 & 205 & 0.7 & \\
\hline$\|$ & 567 & 32 & 9,606 & 30 & .03 & 1,858 & 32 & 8,315 & 30 & \\
\hline III & 1,184 & 67 & 21,962 & 69 & & 3,933 & 68 & 19,213 & 69 & \\
\hline
\end{tabular}




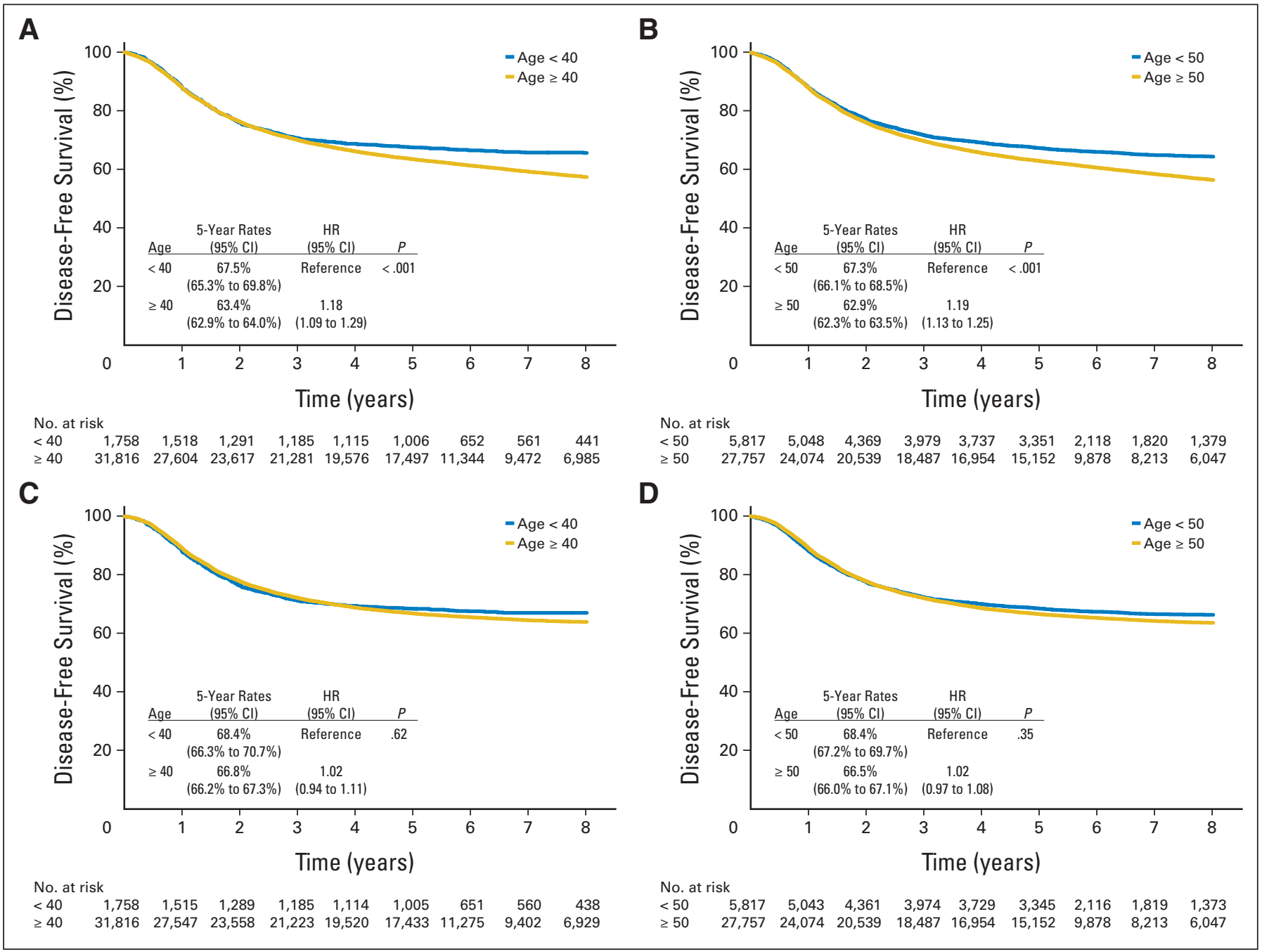

Fig 1. Disease-free survival and time to recurrence for all studies. (A) Disease-free survival, age cutoff 40 years; (B) disease-free survival, age cutoff 50 years; (C) time to recurrence, age cutoff 40 years; (D) time to recurrence, age cutoff 50 years. HR, hazard ratio.

patients were observed when age younger than 50 years was used as the cutoff point to define younger patients.

Based on data from all trials, DFS was significantly longer among younger patients (Figs 1A and 1B) compared with older patients, adjusting for sex and stage. Patients younger than 40 years had a 5-year DFS rate of $67.5 \%$ versus $63.4 \%$ for patients $\geq 40$ years (hazard ratio [HR], $1.18 ; 95 \% \mathrm{CI}, 1.09$ to $1.29 ; P<.001)$. When young patients were defined as being younger than 50 years, the 5 -year DFS rate was $67.3 \%$ and $62.9 \%$ among younger and older patients, respectively (HR, 1.19; 95\% CI, 1.13 to $1.25 ; P<.001)$.

As illustrated in Figures 1C and 1D, the RFI did not differ significantly between older and younger patients. When 40 years was used as the cutoff point, 5-year RFI rate was $68.4 \%$ for younger patients and $66.8 \%$ for older patients (HR, 1.02; 95\% CI, 0.94 to $1.11 ; P=.62$ ). When 50 years was used as the cutoff point, the 5 -year RFI rate was $68.4 \%$ and $65.5 \%$ for younger and older patients, respectively (HR, 1.02 ; $95 \%$ CI, 0.97 to 1.08 ; $P=.35$ ).

When data from all trials were evaluated, OS differed significantly between younger and older patients in both analyses. Patients younger than 40 years had a 5-year OS rate of $75.1 \%$ versus $71.2 \%$ for patients $\geq 40$ years (HR, $1.31 ; 95 \% \mathrm{CI}, 1.20$ to $1.44 ; P<.001$ ). When patients were grouped by younger than 50 years or $\geq 50$ years, the younger group had a 5-year OS rate of 75.5\% compared with 70.5\% for the older patients (HR, 1.33; 95\% CI, 1.26 to $1.40 ; P<.01$ ). When compared with the age-matched expected 5- and 8-year survival rates among the United States white population, the decline in survival in patients younger than 50 years (relative 5-year survival rate, $77.0 \%$; 8 -year survival rate, $72.4 \%$ ) was similar to that in patients $\geq 50$ years (relative 5-year survival rate, 78.5\%; 8-year survival rate, 74.4\%; Fig 2).

The effect of age was tested separately for stage II and III patients. A significant interaction was observed for DFS (age $<40$ years, $P=.004$; age $<50$ years, $P<.001)$ and OS $(P<.001$ for both age $<40$ and age $<50$ years) between age group and stage. Specifically, a larger impact of age group on both DFS and OS was observed for stage II patients compared with stage III patients. For DFS, the HR comparing older with younger patients was larger in stage II patients (age $<40$ years: HR, 1.51; 95\% CI, 1.24 to 1.84 ; $P<.01$; age $<50$ years: HR, $1.52 ; 95 \% \mathrm{CI}, 1.38$ to $1.70 ; P<.01)$ than that in stage III patients (age $<40$ years: HR, 1.11; 95\% CI, 1.02 to $1.22 ; P=.02$; age $<50$ years: $\mathrm{HR}, 1.13$; $95 \% \mathrm{CI}, 1.07$ to 1.19 ; 


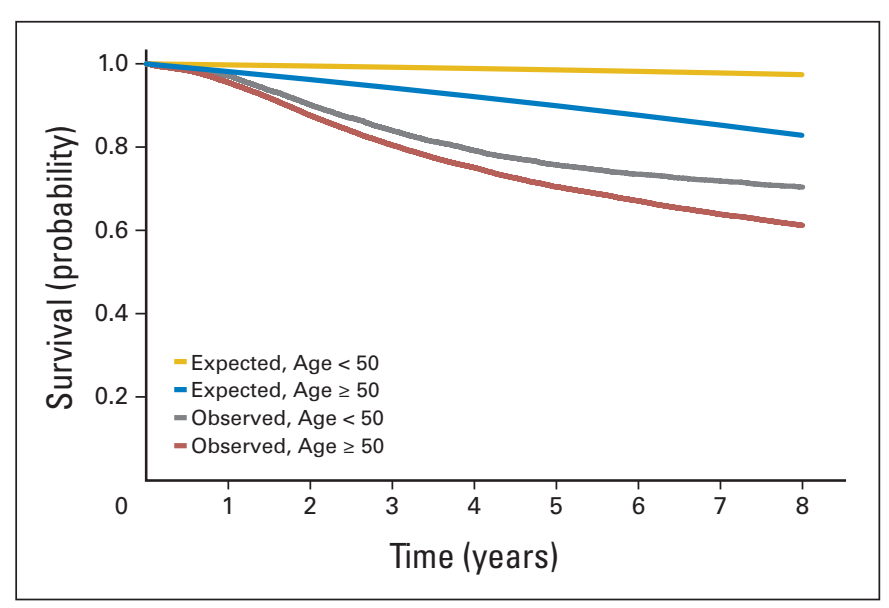

Fig 2. Observed versus expected survival based on United States white population.

$P<.01)$. Similarly, the OS HR comparing older with younger patients is larger in stage II patients (age $<40$ years: $\mathrm{HR}, 1.84 ; 95 \%$ CI, 1.47 to $2.30 ; P<.01$; age $<50$ years: $\mathrm{HR}, 1.81 ; 95 \% \mathrm{CI}, 1.60$ to $2.04 ; P<.01$ ) than for stage III patients (age $<40$ years: $\mathrm{HR}, 1.21$; 95\% CI, 1.09 to $1.33 ; P<.01$; age $<50$ years: $\mathrm{HR}, 1.24 ; 95 \% \mathrm{CI}$, 1.17 to $1.31 ; P<.01)$.

When DFS analyses were restricted to the nine studies that demonstrated a benefit to adjuvant therapy, younger patients derived a benefit similar to older patients. For DFS, the HR comparing adjuvant treatment versus control or oxaliplatin plus FU/LV versus FU/LV alone was 0.76 for patients younger than 40 years (95\% CI, 0.51 to $1.0 ; P=.05$ ) and 0.75 for patients $\geq 40$ years ( $95 \%$ CI, 0.69 to $0.80 ; P<.001$; interaction $P=0.78$; Figs $3 \mathrm{~A}$ and $3 \mathrm{~B})$. The DFS HR for therapeutic benefit was 0.66 (95\% CI, 0.54 to $0.80 ; P<.001)$ and $0.76(95 \% \mathrm{CI}, 0.70$ to $0.82 ; P<.001)$ for patients younger than 50 years and $\geq 50$ years, respectively (interaction $P=0.19$; Figs $3 \mathrm{C}$ and $3 \mathrm{D}$ ).

OS within the nine trials with adjuvant treatment benefit was similar between patients younger than 50 years and $\geq 50$ years in the treatment arms for each group (HR, 0.79; 95\% CI, 0.63 to 0.98 ; $P=.03$; and HR, 0.79; 95\% CI, 0.73 to $0.87 ; P<.001$, respectively; interaction $P=0.97$ ). For those patients younger than 40 years, 5 -year OS was $78.8 \%$ in the experimental arms and $78.2 \%$ in the control arm. The HR for treatment benefit was 0.94 (95\% CI, 0.64 to $1.38 ; P=.74)$. For patients $\geq 40$ years, 5 -year OS was $76.6 \%$ in the treatment arms and $72.4 \%$ in the control arms (HR, 0.79; 95\% CI, 0.73 to $0.86 ; P<.001)$. Although the HRs appear numerically different, the interaction $P$ value was 0.49 , providing no evidence of a differential effect. These findings held true in subgroup analyses restricted to patients with stage III disease alone.

The occurrence of grade $\geq 3$ AEs were analyzed by age group (Table 3 ). When stratified by age younger than 40 years, younger patients had less leukopenia $(3 \% v 8 \% ; P=.02)$ and more nausea and vomiting $(10 \% \vee 7 \% ; P=.04)$, adjusting for sex and stage. In the $\mathrm{AE}$ analysis with age 50 years as the cutoff, the decreased incidence of leukopenia remained significant $(P=.007)$. In addition, patients 50 years or older had significantly increased rates of stomatitis $(10 \%)$ compared with those younger than 50 years $(6 \%$; $P=.003)$.

\section{DISCUSSION}

This analysis of the ACCENT database demonstrates that younger patients with stage II and III colon cancer, defined as either younger than 40 years or younger than 50 years, derive similar benefit from adjuvant therapy as older patients in terms of DFS and RFI.

Because the ACCENT database consists of data from randomized trials, we were able to compare the treatment benefit of adjuvant therapy between younger and older patients. The improvements in DFS were similar in all age groups when the analysis was restricted to the nine clinical trials with benefit to adjuvant therapy for both age cutoff points.

Among all clinical trials, younger patients had improved DFS and OS compared with older patients. The RFI was similar among the age groups, indicating the survival benefit for younger patients is likely related to fewer competing causes of death. Indeed, the relative survival rates, when compared with the respective United States population, are similar between older and younger patients. The findings of this study are comparable to results of other analyses showing that stage by stage younger patients have similar to improved survival rates when compared with older patients. ${ }^{13,14}$

The finding that age is a more important prognostic factor in patients with stage II and III disease has strong biologic rationale. Recurrence rates for patients with stage II disease are low (10\% to $20 \%),{ }^{15}$ thus most mortality in the stage II population is as a result of competing causes, which are highly age dependent. Stage III patients are more likely to have a disease-related death (recurrence rates of approximately $30 \%),{ }^{16}$ which decreases the impact of deaths as a result of noncancer causes.

The suggestion of possible lack of OS benefit for adjuvant therapy for patients younger than 40 years also has biologic plausibility. It is well established that tumor biology in young adults differs from that of older adults. ${ }^{15}$ Younger patients have higher rates of high microsatellite instability (MSI-H), a finding associated with higher rates of survival when compared with patients with microsatellite stable tumors in untreated or FU-based adjuvant therapy-treated patients. ${ }^{16}$ The younger-than-40-years population in our study may have included a higher rate of MSI-H tumors, improving survival rates regardless of the impact of adjuvant therapy. In addition, there is evidence that patients with MSI-H tumors do not benefit from FU-based adjuvant therapy (acknowledging that this evidence is strongest in sporadic versus germline MSI-H tumors ${ }^{17}$ ). The elevated survival and lack of adjuvant therapy benefit in MSI-H tumors potentially explain the lack of OS benefit from adjuvant therapy in the younger-than-40-years age group. However, because a significant benefit was observed for RFI and DFS, the OS interaction $P$ value was not significant, and because the number of patients younger than 40 years in the treatment benefit analysis was quite modest, this may be a chance finding.

One limitation of our study was the small percentage of patients younger than 30 years. A subset of 20 - to 40 -year-old patients present with more aggressive, advanced disease contributing to poorer outcomes at the youngest end of the spectrum. ${ }^{18}$ This is felt to be another subset of tumor biology more frequent in the youngest adult patients. Chiang et al ${ }^{19}$ found the proportion of patients with mucin-producing tumors, a characteristic associated with more aggressive tumor behavior, was greater in patients younger than 30 years and decreased with patient age. This phenotype, which is felt to be a major contributor to 


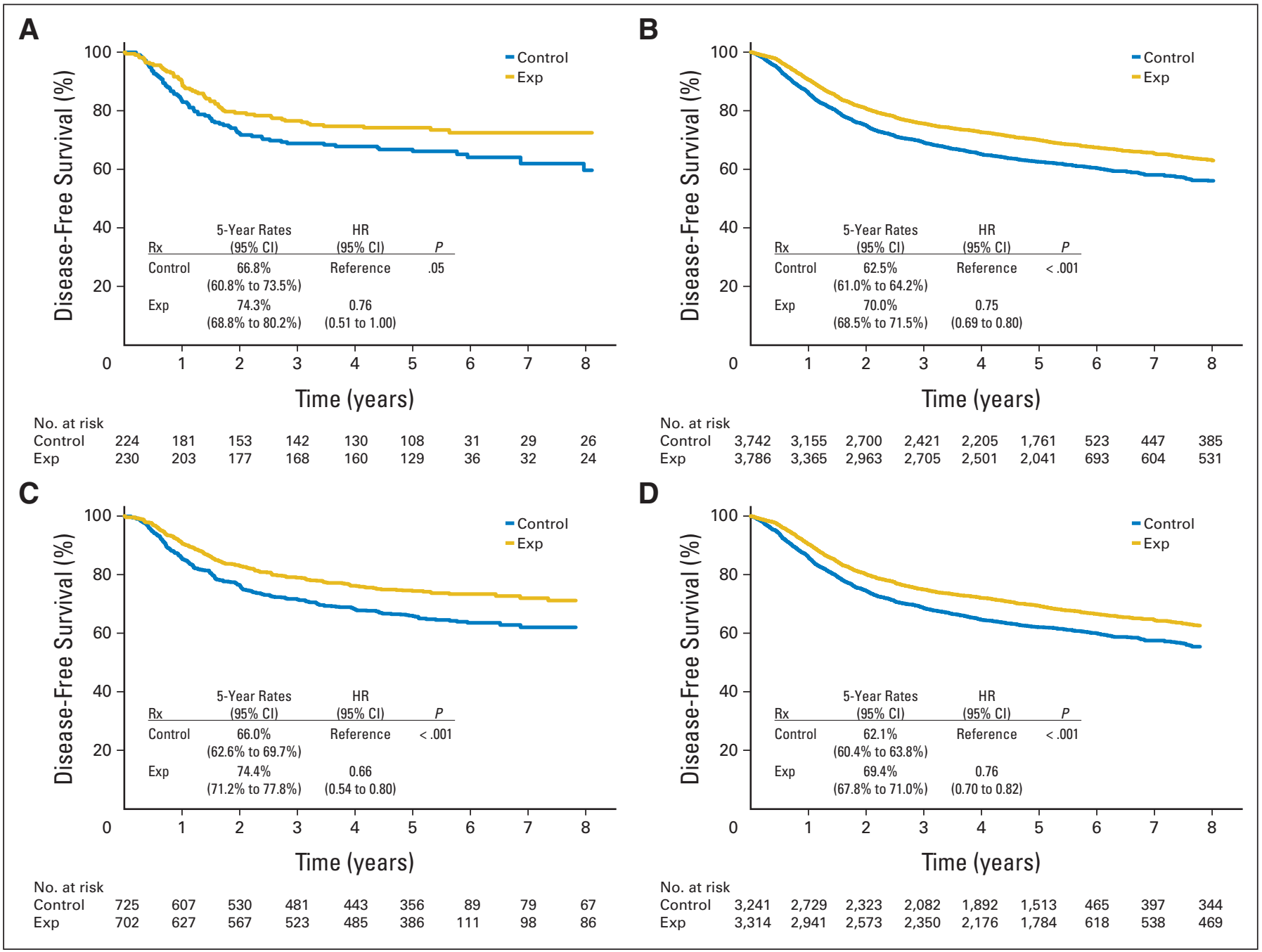

Fig 3. Disease-free survival: experimental (Exp) versus control. (A) Age $<$ than 40 years; (B) age $\geq 40$ years; (C) age $<50$ years; (D) age $\geq 50$ years. HR, hazard ratio; $R x$, treatment.

mortality of young adults with advanced colon cancer, was likely underrepresented in this analysis.

The AYAO PRG has also reported on the more aggressive clinical behavior of colorectal cancers in adolescents and young adults. ${ }^{6}$ Potential biologic differences contributing to these observations include a higher incidence of genetic susceptibility syndromes, mutations in the TGFBR2 gene, higher frequency of mucinous adenocarcinoma, and higher rates of signet ring histology. Future studies will focus on the definitive determination of molecular differences between adolescent and young adult patients and older adult patients and investigate whether these differences explain the natural history of colon cancer in the youngest adults or predict response to treatment.

On the basis of the adverse events analysis within the 10 clinical trials with toxicity data, younger patients seem to tolerate therapy just as well, if not better, than older patients. An exception was nausea and vomiting, of which younger patients had a modestly higher incidence

\begin{tabular}{|c|c|c|c|c|c|c|c|c|c|c|}
\hline Leukopenia & 3 & 8 & 2.82 & 1.12 to 4.66 & .02 & 4 & 8 & 1.89 & 1.30 to 2.74 & .007 \\
\hline Stomatitis & 6 & 9 & 1.71 & 0.97 to 3.01 & .06 & 6 & 10 & 1.60 & 1.17 to 2.17 & .003 \\
\hline Nausea/vomiting & 10 & 7 & 0.64 & 0.42 to 0.98 & .04 & 8 & 7 & 0.80 & 0.60 to 1.05 & .11 \\
\hline
\end{tabular}


of grade 3 toxicity. Particular attention to nausea and vomiting, including more aggressive use of prophylactic antiemetic regimens, is warranted in younger adults to minimize this adverse effect and promote compliance.

In conclusion, patients younger than 50 years with stage II and III colon cancer enrolled onto clinical trials had similar benefits from adjuvant therapy as older patients. It has been recognized that this population is less likely to be insured and use medical resources, leading to a delay in diagnosis. ${ }^{20}$ The potential for high survival with stage II and III colon cancer stresses the importance of early recognition and appropriate treatment of this population.

\section{AUTHORS' DISCLOSURES OF POTENTIAL CONFLICTS OF INTEREST}

Although all authors completed the disclosure declaration, the following author(s) indicated a financial or other interest that is relevant to the subject matter under consideration in this article. Certain relationships marked with a " $U$ " are those for which no compensation was received; those relationships marked with a " $C$ " were compensated. For a detailed description of the disclosure categories, or for more information about ASCO's conflict of interest policy, please refer to the Author Disclosure
Declaration and the Disclosures of Potential Conflicts of Interest section in Information for Contributors.

Employment or Leadership Position: None Consultant or Advisory Role: Archie Bleyer, Sigma-Tau Pharmaceuticals (C) Stock Ownership: None Honoraria: Archie Bleyer, Sigma-Tau Pharmaceuticals Research Funding: None Expert Testimony: None Other Remuneration: None

\section{AUTHOR CONTRIBUTIONS}

Conception and design: David M. Thomas, Charles Blanke, Daniel Sargent

Financial support: Daniel Sargent

Administrative support: Charles Blanke, Michael J. O'Connell, Daniel Sargent

Provision of study materials or patients: Greg Yothers, Roberto

Labianca, Daniel Sargent

Collection and assembly of data: Greg Yothers, Charles Blanke, Roberto Labianca, Aimery de Gramont, Daniel Sargent

Data analysis and interpretation: Joleen Hubbard, David M. Thomas, Greg Yothers, Erin Green, Charles Blanke, Michael J. O'Connell, Qian Shi, Archie Bleyer, Aimery de Gramont, Daniel Sargent

Manuscript writing: All authors

Final approval of manuscript: All authors

\section{REFERENCES}

1. Jemal $A$, Siegel $R, X u$ J, et al: Cancer statistics, 2010. CA Cancer J Clin 60:277-300, 2010

2. Bleyer $A$, Choi $M$, Fuller $C D$, et al: Relative lack of conditional survival improvement in young adults with cancer. Semin Oncol 36:460-467, 2009

3. Bleyer A, Budd T, Montello M: Adolescents and young adults with cancer: The scope of the problem and criticality of clinical trials. Cancer 107: 1645-1655, 2006

4. Bleyer A: The adolescent and young adult gap in cancer care and outcome. Curr Probl Pediatr Adolesc Health Care 35:182-217, 2005

5. National Cancer Institute, and the LiveStrong Young Adult Alliance: Closing the Gap: Research and Care Imperatives for Adolescents and Young Adults with Cancer Report of the Adolescent and Young Adult Oncology Progress Review Group: Report of the Young Adult Oncology Progress Review Group. Bethesda, MD, National Cancer Institute and the Livestrong Young Adult Alliance, NIH Publication No. 06-6067, 2006

6. Tricoli JV, Seibel NL, Blair DG, et al: Unique characteristics of adolescent and young adult acute lymphoblastic leukemia, breast cancer, and colon cancer. J Natl Cancer Inst 103:628-635, 2011
7. Bleyer A: Young adult oncology: The patients and their survival challenges. CA Cancer J Clin 57:242-255, 2007

8. $\mathrm{O}^{\prime}$ Connell JB, Maggard MA, Liu JH, et al: Do young colon cancer patients have worse outcomes? World J Surg 28:558-562, 2004

9. Zbuk K, Sidebotham EL, Bleyer A, et al: Colorectal cancer in young adults. Semin Oncol 36:439450, 2009

10. Sargent DJ, Wieand HS, Haller DG, et al: Disease-free survival versus overall survival as a primary end point for adjuvant colon cancer studies: Individual patient data from 20,898 patients on 18 randomized trials. J Clin Oncol 23:8664-8670, 2005

11. Sargent D, Shi Q, Yothers G, et al: Two or three year disease-free survival (DFS) as a primary end-point in stage III adjuvant colon cancer trials with fluoropyrimidines with or without oxaliplatin or irinotecan: Data from 12,676 patients from MOSAIC, X-ACT, PETACC-3, C-06, C-07 and C89803. Eur J Cancer 47:990-996, 2011

12. Chansky K, Benedetti J, Macdonald JS: Differences in toxicity between men and women treated with 5-fluorouracil therapy for colorectal carcinoma. Cancer 103:1165-1171, 2005

13. O'Connell JB, Maggard MA, Liu JH, et al: Are survival rates different for young and older patients with rectal cancer? Dis Colon Rectum 47:20642069, 2004
14. O'Connell JB, Maggard MA, Livingston EH, et al: Colorectal cancer in the young. Am J Surg 187:343-348, 2004

15. Bleyer A, Barr R, Hayes-Lattin B, et al: The distinctive biology of cancer in adolescents and young adults. Nat Rev Cancer 8:288-298, 2008

16. Gryfe R, Kim H, Hsieh ET, et al: Tumor microsatellite instability and clinical outcome in young patients with colorectal cancer. N Engl J Med 342: 69-77, 2000

17. Sinicrope FA, Foster NR, Thibodeau SN, et al: DNA mismatch repair status and colon cancer recurrence and survival in clinical trials of 5-fluorouracil-based adjuvant therapy. J Natl Cancer Inst 103:863-875, 2011

18. LaQuaglia MP, Heller G, Filippa DA, et al: Prognostic factors and outcome in patients 21 years and under with colorectal carcinoma. J Pediatr Surg 27:1085-1089, 1992 (discussion 1889-1890)

19. Chiang JM, Chen MC, Changchien CR, et al: Favorable influence of age on tumor characteristics of sporadic colorectal adenocarcinoma: Patients 30 years of age or younger may be a distinct patient group. Dis Colon Rectum 46:904-910, 2003

20. Martin S, Ulrich C, Munsell M, et al: Delays in cancer diagnosis in underinsured young adults and older adolescents. Oncologist 12:816-824, 2007 